\title{
Erratum to: Static analysis of variable thickness two-directional functionally graded annular sector plates fully or partially resting on elastic foundations by the GDQ method
}

Farhad Alinaghizadeh $^{1} \cdot$ Mahmoud Shariati $^{1}$

Published online: 8 February 2016

(C) The Brazilian Society of Mechanical Sciences and Engineering 2016

Erratum to: J Braz. Soc. Mech. Sci. Eng. (2015)

\section{7:1819-1838}

DOI 10.1007/s40430-015-0427-0

The original version of this article unfortunately contained a mistake.

The presentation of Eq. 7 was incorrect. The correct version is given below.

$z_{0}=\frac{h(r) n_{\mathrm{z}}}{2\left(n_{\mathrm{z}}+2\right)} \frac{\left(E_{\mathrm{c} 2}-E_{\mathrm{m} 2}\right)+\left(\left(E_{\mathrm{c} 1}-E_{\mathrm{m} 1}\right)-\left(E_{\mathrm{c} 2}-E_{\mathrm{m} 2}\right)\right)\left(\frac{r-r_{\mathrm{i}}}{r_{\mathrm{o}}-r_{\mathrm{i}}}\right)^{n_{\mathrm{r}}}}{\left(E_{\mathrm{c} 2}+n_{\mathrm{z}} E_{\mathrm{m} 2}\right)+\left(E_{\mathrm{c} 1}-E_{\mathrm{c} 2}+n_{\mathrm{z}}\left(E_{\mathrm{m} 1}-E_{\mathrm{m} 2}\right)\right)\left(\frac{r-r_{\mathrm{i}}}{r_{\mathrm{o}}-r_{\mathrm{i}}}\right)^{n_{\mathrm{r}}}}$.

The online version of the original article can be found under doi:10.1007/s40430-015-0427-0.

Mahmoud Shariati

mshariati44@um.ac.ir; mshariati44@gmail.com

Farhad Alinaghizadeh

farhad.alinaghi.zadeh@gmail.com

1 Department of Mechanical Engineering, Faculty

of Engineering, Ferdowsi University of Mashhad, Mashhad,

Iran 